\title{
Prélèvements et transferts sociaux : une analyse descriptive des incitations financières au travail
}

Guy Laroque et Bernard Salanié*

* Guy Laroque est directeur des Études et synthèses économiques de l'Insee, Bernard Salanié est chef de la division Croissance et politiques macroéconomiques de l'Insee. Les auteurs ont bénéficié de conversations avec Cédric Afsa, Alain Bayet, François Bourguignon, François Brunet, Anne Flipo, Denis Fougère, Anne Laferrère, Christine Lagarenne, Sylvie Le Minez, Stefan Lollivier, Jean-Paul Lorgnet, Thierry Magnac, Pierre Morin, Thomas Piketty, Jean-Marc Robin, Nicole Roth, Christophe Starzec, Daniel Verger, qui ne sont pas responsables des erreurs qui subsistent. IIs ont également profité des commentaires de trois lecteurs anonymes.
Les noms et dates entre parenthèses renvoient à la bibliographie en fin d'article.

Un ensemble complexe de prélèvements et de transferts sociaux s'interpose entre la rémunération versée aux ménages et le revenu dont ils disposeront effectivement. D'un côté, cotisations sociales, impôts et taxes viennent grever ce revenu ; de l'autre, prestations sociales et allocations l'augmentent. Mais le fonctionnement de ce système a des conséquences variables sur le niveau du revenu disponible d'un ménage en fonction des caractéristiques de ce ménage (situation du conjoint, nombre d'enfants) et du niveau de ses revenus (RMI, bas salaires). Jusqu'à présent, ce fonctionnement n'était décrit qu'à travers l'analyse de cas-types. $L$ 'application de ce système à un échantillon représentatif d'une partie de la population française (près de 20 millions d'individus) permet, en plus, d'étudier la répartition des taux nets de prélèvement dans cette sous-population.

Des exercices de simulation réalisés, il ressort que ce sont les ménages ayant les revenus les plus bas qui ont les taux marginaux de prélèvement les plus hauts, ce qui peut avoir pour effet de limiter les effets des incitations financières à la reprise d'un emploi. En particulier, l'incitation financière à reprendre un emploi payé au Smic paraît faible pour nombre des chômeurs et des inactifs.

E ntre les rémunérations que les employeurs versent aux ménages et leur revenu disponible s'interpose un ensemble complexe de prélèvements et de transferts, résultat de ce qu'on peut appeler le «système socio-fiscal ». Ainsi, les chômeurs peuvent bénéficier de l'allocation unique dégressive (AUD) pendant un temps limité, de l'allocation de solidarité spécifique (ASS) ou du revenu minimum d'insertion (RMI). Par ailleurs, les ménages élevant des enfants peuvent prétendre à des prestations familiales diverses. En ce qui concerne les prélèvements, la moitié des foyers fiscaux français paient l'impôt sur le revenu et la plupart s'acquittent de la taxe d'habitation. Enfin, les rémunérations versées par les employeurs sont amputées de cotisations sociales qui, pour être moins visibles que l'impôt sur le revenu, n'en doivent pas moins être analysées comme des prélèvements sur les revenus du travail.

Le fonctionnement du système socio-fiscal a pour conséquence qu'une augmentation donnée de la rémunération versée à un individu peut avoir des effets très variables sur le revenu disponible du ménage auquel il appartient suivant les caractéristiques de ce ménage et le niveau de ses revenus. En règle générale, une telle augmentation aboutira à augmenter le revenu disponible du ménage d'un montant inférieur. Ceci s'analyse comme une désincitation au travail, dont certains pensent qu'elle est devenue très forte pour les hauts revenus. En fait, l'analyse du système socio-fiscal 
montre que c'est au bas de l'échelle que ses effets sont les plus importants. Ainsi, pour les RMIstes, une augmentation de la rémunération conduit à une augmentation du revenu disponible du ménage souvent nulle et qui peut même devenir négative, la sortie du RMI s'accompagnant de la perte d'une partie des allocations logement et d'une augmentation de la taxe d'habitation. La situation des RMIstes, en particulier, a parfois soulevé la crainte que leur incitation à reprendre un travail soit trop faible. C'est ce qu'on appelle souvent la « trappe à pauvreté » ou la «trappe à inactivité ».

Encadré 1

\section{UNE SIMULATION SUR L'ENQUÊTE EMPLOI}

L'Insee réalise tous les ans une enquête Emploi auprès d'un échantillon représentatif de ménages. Cette enquête comprend de nombreuses questions sur les caractéristiques du ménage, sa situation vis-à-vis de l'emploi et ses salaires éventuels. Cet article utilise l'enquête Emploi de mars 1997.

\section{Une modélisation des prestations et des prélèvements...}

Dans un premier temps, les barèmes légaux ou réglementaires ont été utilisés pour modéliser les divers prestations et prélèvements aussi précisément que possible : cotisations sociales à la charge des employeurs et des salariés, prestations familiales (allocations familiales, complément familial, allocation pour jeune enfant, allocation parentale d'éducation, allocation de parent isolé, allocation de rentrée scolaire, aides à la scolarité), allocations logement, RMI et taxe d'habitation. On n'a pas tenu compte des aides accordées par les collectivités locales. Dans un deuxième temps, l'effet du système socio-fiscal ainsi défini est simulé sur tous les ménages interrogés dans l'enquête.

Cependant, les données de l'enquête ne permettent pas toujours de reconstituer les prestations ou prélèvements théoriques. Certaines catégories de ménages ont dû être laissées de côté et des approximations souvent fortes ont été faites. L'enquête Emploi ne donne pas d'indications sur les transferts entre ménages, ce qui est particulièrement gênant pour les femmes divorcées, dont beaucoup bénéficient de pensions alimentaires. Plus grave, elle ne renseigne pas non plus sur les revenus financiers ou immobiliers des ménages (1), sur les retraites, ou sur les revenus des ménages dont un membre est employeur ou travailleur indépendant. Aussi, les résultats contenus dans cet article ne sont-ils pas représentatifs de l'ensemble de la population française : ils concernent les salariés et les chômeurs, mais n'intègrent pas bien les titulaires de hauts revenus.

\section{... dans une optique de long terme}

L'optique choisie est de long terme : le calendrier des prélèvements et transferts du système fiscalo-social est important mais très complexe, comme le montrent sur des cas-types Paillaud et Eyssartier (1999). Ainsi, les provisions d'intéressement qui permettent à un RMlste de conserver son allocation pendant une courte période après la reprise d'un emploi sont négligées, tout comme les allocations chômage. Celles-ci sont pour l'essentiel de deux types. L'allocation unique dégressive (AUD) dépend du salaire passé et est versée à un taux décroissant pendant une période limitée. En 1997, l'allocation relevait, pour plus de la moitié des chômeurs indemnisés, de la « filière 5 ». Ces chômeurs ont moins de 50 ans et ont travaillé au moins 14 mois durant les deux ans précédant la fin de leur contrat de travail. Ils ont droit pendant 9 mois à l'AUD au taux plein (limité à $75 \%$ du salaire de référence), puis pendant 21 mois à l'AUD au taux dégressif (avec réduction de $17 \%$ tous les 6 mois). La reprise d'un travail interrompt le versement de I'AUD, sauf si le nouveau salaire est inférieur de plus de $30 \%$ à l'ancien. Dans ce cas, la personne concernée peut conserver le bénéfice d'une AUD réduite, multipliée par (1 - nouveau salaire/ancien salaire), pendant une durée maximale de 18 mois. Étant donné l'optique de long terme retenue, l'AUD n'est pas simulée (2).

En revanche, nous aurions souhaité modéliser l'allocation de solidarité spécifique (ASS). En effet, l'ASS prend le relais de l'AUD en fin de droits et peut, en pratique, être versée jusqu'à la retraite si le ménage continue à remplir les conditions de ressources exigées. L'ASS est d'environ 2300 francs par mois quand les ressources du ménage sont inférieures à 2900 francs par mois pour une personne seule, 5900 francs pour un couple. Au-dessus de ces seuils, l'ASS devient une allocation différentielle qui amène les ressources du ménage respectivement à 5200 francs par mois pour une personne seule et à 8200 francs pour un couple (3). Malheureusement, les informations présentes dans l'enquête Emploi sur les allocations chômage ne permettent pas de distinguer les sommes perçues au titre de l'ASS. Pour les ménages dont les ressources sont supérieures au plafond de versement de l'ASS, cette convention est sans conséquence. Pour les autres, la substitution du RMI à l'ASS a comme effet d'augmenter les taux de prélèvement pour les ménages dont les ressources sont inférieures au montant de l'ASS (le taux marginal simulé est de $100 \%$, alors qu'il est égal en fait au taux qui résulte de l'application des barèmes de cotisations sociales, CSG et CRDS, allocation logement, etc.) et, réciproquement, de les minorer lorsque les ressources du ménage sont comprises entre le RMI et le plafond de l'ASS.

1. Nous ne disposons donc pas d'estimation fiable des taux de prélèvement supportés par les plus riches.

2. La différence entre le taux de prélèvement à court terme et le taux à long terme calculé dépend de la situation de la personne et du ménage dont elle fait partie. Pour un ménage avec de faibles ressources salariales, le long terme est le RMI, avec son caractère différentiel (taux marginal de $100 \%$ en dessous du plafond) alors que le taux marginal à court terme est égal au rapport de l'AUD à l'ancien salaire, nettement inférieur à 1. Pour un ménage disposant de ressources élevées, le taux de court terme est égal au taux de long terme plus le rapport de l'AUD à l'ancien salaire.

3. Comme pour l'AUD et le RMI, il y a un mécanisme d'intéressement qui réduit, sans la supprimer complètement, l'ASS pour les 750 premières heures ouvrées lors de la reprise d'un emploi. 
L'analyse des désincitations au travail et des éventuelles trappes à pauvreté peut procéder selon trois étapes successives. La première consiste à étudier le fonctionnement du système socio-fiscal sur des cas-types : on s'intéresse, par exemple, à un couple monoactif élevant un jeune enfant et on analyse les variations du revenu disponible de ce ménage en fonction de la rémunération de son membre actif. L'article de Paillaud et Eyssartier (1999), qui expose les résultats obtenus à la direction de la Prévision à l'aide de la maquette Pâris, en est un bon exemple. L'étude de cas-types permet de rapprocher les divers éléments du système, souvent conçus indépendamment les uns des autres, et d'examiner leurs interactions. Elle fait apparaître des trappes à pauvreté potentielles, mais elle ne permet pas d'évaluer le nombre de ménages français qui se trouvent effectivement dans ces situations. La deuxième étape consiste donc à appliquer le système socio-fiscal à un échantillon de la population française, afin d'étudier, en quelque sorte, la répartition des incitations au travail dans la population ; c'est ce qui est présenté dans cet article (1).

On remarquera qu'à ce stade, la mise en évidence de trappes à pauvreté, si elle se confirme, n'implique pas jugement quant à leur effet sur les comportements des individus concernés. Ceux-ci peuvent désirer travailler, quel que soit le salaire offert, pour satisfaire un désir d'intégration sociale par exemple. Il faut comparer rémunération du travail et " salaire de réservation », salaire à partir duquel la personne est prête à travailler. Ce salaire de réservation dépend, entre autres, des caractéristiques du ménage, en particulier du nombre d'enfants en bas âge. La troisième étape procède donc à l'estimation économétrique de ce salaire de réservation afin d'évaluer l'effet des trappes à pauvre té sur les comportements de participation (2). Des résultats obtenus par cette approche sont présentés dans Laroque et Salanié (1999a ; 1999b).

La base statistique de cette étude est l'enquête Emploi de mars 1997. Toutefois, cette enquête ne permet pas de retenir dans les calculs les revenus financiers ou immobiliers, les retraites et les allocations chômage. On se place, par ailleurs, dans une perspective de long terme (cf. encadré 1).

\section{Trois exercices de simulation}

Nous avons procédé à trois exercices. Le premier consiste à calculer les taux de prélèvements marginaux auxquels sont confrontés les ménages et leur distribution dans la population étudiée. Le second vise à aller au-delà du marginal : évaluer l'accroissement du coût du travail pour l'employeur garantissant une hausse de revenu disponible de 1000 francs pour le ménage. Enfin, le dernier s'intéresse à la trappe à inactivité proprement dite : il examine l'incitation financière que les chômeurs ou les inactifs ont à reprendre un emploi, à mi-temps ou à temps plein, rémunéré au Smic.

La mise en place de minima sociaux, qui assurent aux plus démunis un plancher de ressources, a pour contrepartie, lorsque les revenus d'activité s'élèvent et les prestations sous conditions de ressources disparaissent, une zone affichant des taux marginaux de prélèvement très forts. Ceci fait courir le risque de voir la population scindée en deux groupes, l'un bénéficiant des aides sociales, l'autre au travail, avec peu de mouvements d'un groupe à l'autre en raison de la structure du système socio-fiscal. L'étude fournit des indications chiffrées sur ce phénomène. Sur les 20 millions de personnes de moins de 55 ans qui appartiennent à des ménages sans retraités, employeurs ou travailleurs indépendants, un peu moins de $20 \%$, soit près de 4 millions d'individus, seraient soumis à un taux de prélèvement marginal supérieur à $95 \%$. Sur les 5 millions de chômeurs et inactifs, plus de $30 \%$ verraient leurs ressources nettes s'accroître de moins de 2000 francs par mois s'ils prenaient un emploi à plein temps au Smic. Cet accroissement de ressources est bien sûr considérable pour la population concernée, mais il reste inférieur à $40 \%$ du Smic net.

\section{Les imputations}

$\mathrm{L}$ 'enquête Emploi fournit notamment les salaires nets reçus par les membres du ménage, la durée hebdomadaire de leur travail, l'âge et l'état matrimonial, le nombre et l'âge des enfants, des caractéristiques du logement (notamment s'il s'agit d'une HLM, s'ils sont propriétaires ou locataires). Sur la base de ces informations, sont estimés successivement le coût du travail pour l'employeur des

1. Certains travaux de ce type ont déjà été réalisés en France ou à l'étranger. Ils se limitent souvent à l'impôt sur le revenu ((Lang et Nohrbass-Stahl, 1997) pour l'Allemagne, (Mercader et Prats, 1997) pour l'Espagne ou (Hakkio et Rush-Schmidt, 1996) pour les États-Unis). L'article de Bourguignon, Chiappori et Hugounenq (1993), pour la France, présente des résultats plus globaux tirés de l'exploitation de l'enquête Budget de Famille ; ces résultats, peu détaillés, concernent l'année 1991 et ne retracent qu'une partie du système socio-fiscal.

2. II existe d'autres approches de ce problème. Ainsi, Piketty (1998) estime l'impact de l'extension de l'allocation parentale d'éducation aux mères de deux enfants en comparant l'évolution de leur taux d'emploi à un groupe de contrôle formé de mères non affectées par cette mesure. 
personnes interrogées, leur revenu imposable et l'impôt sur le revenu des personnes physiques dont elles sont redevables, les prestations familiales auxquelles elles ont droit, les allocations loge-

Tableau 1
$\begin{aligned} & \text { Le champ couvert : près de } 20 \text { millions } \\
& \text { d'individus }\end{aligned}$
\begin{tabular}{|l|c|c|}
\hline & $\begin{array}{c}\text { Total } \\
\text { enquête }\end{array}$ & $\begin{array}{c}\text { Champ de } \\
\text { l'étude }\end{array}$ \\
\hline Nombre de ménages & 23,7 & 14,3 \\
\hline Nombre d'individus & 47,0 & 19,9 \\
dont : Actifs occupés & 22,4 & 14,8 \\
Chômeurs & 3,2 & 2,0 \\
Inactifs & 21,4 & 3,1 \\
\hline
\end{tabular}

Source : calculs des auteurs.

\section{Graphique I}

\section{Prélèvements sociaux}

A - Coût du travail et salaire net

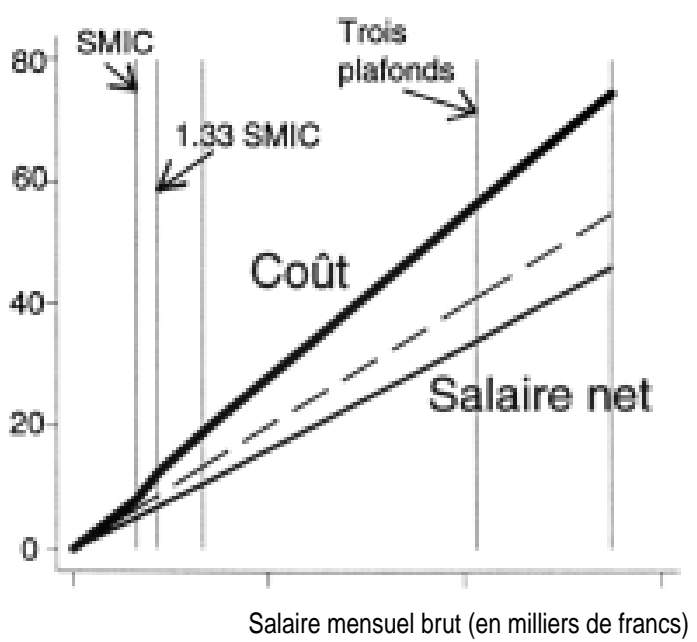

B - Taux marginal de prélèvement sur coût du travail

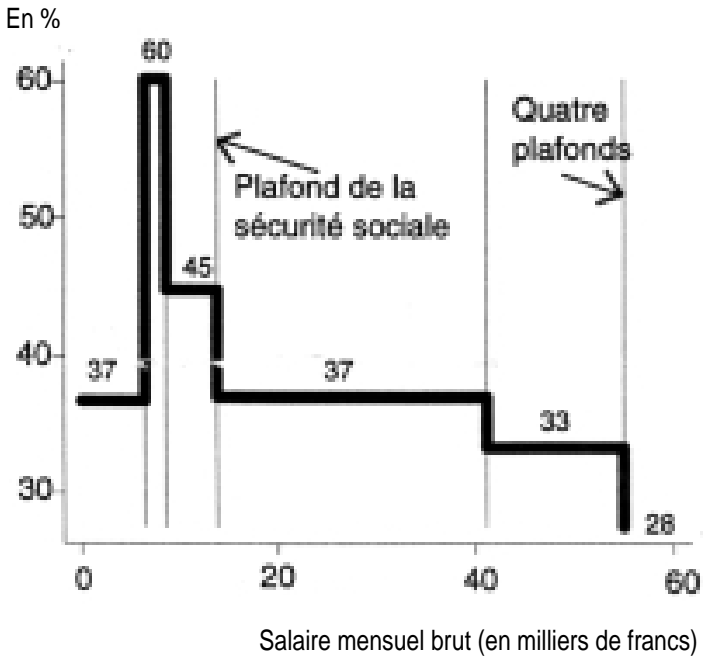

Source : calculs des auteurs. ment et le RMI dont elles peuvent bénéficier et la taxe d'habitation dont elles doivent s'acquitter.

\section{Le champ couvert : près de $\mathbf{2 0}$ millions d'individus}

La non-disponibilité de certaines informations dans l'enquête Emploi conduit à ne retenir qu'un sous-ensemble restreint de la population. Ainsi, l'enquête ne fournit ni les pensions de retraite, ni les revenus des employeurs ou travailleurs indépendants. Sont donc écartés les ménages dont un membre au moins se déclare employeur, travailleur indépendant ou retraité. On passe ainsi des 23,7 millions de ménages de l'enquête à seulement 14,3 millions (cf. tableau 1). Pour comparer les estimations sur barème des prestations familiales aux statistiques agrégées de la Caisse nationale des allocations familiales (CNAF), les calculs sont réalisés à la fois sur l'ensemble de l'enquête et sur l'échantillon restreint ainsi obtenu.

Les personnes âgées de 55 ans ou plus sont écartées en l'absence de renseignements sur les préretraites. Les membres du ménage autres que la personne de référence et son conjoint ne sont pas non plus retenus, dans la mesure où il est difficile de déterminer leurs droits à prestation indépendamment du reste du ménage. On ignore ainsi les étudiants qui habitent chez leurs parents, mais également de nombreux actifs : 3 millions, dont 2,2 millions sont occupés et 0,8 million chômeurs. En définitive, 19,9 millions d'individus sont suivis, qui se décomposent en 14,8 millions d'actifs occupés, 2,0 millions de chômeurs, et 3,1 millions d'inactifs (cf. tableau 1).

\section{Du coût du travail au salaire net}

Le passage du coût du travail au salaire net (et réciproquement) est relativement simple : il s'agit d'une fonction linéaire par morceaux, qui retrace les taux de cotisation employeur et salarié, ainsi que la contribution sociale généralisée (CSG) et la contribution au remboursement de la dette sociale (CRDS), selon les divers plafonds (3). Les barèmes du secteur privé pour les salariés à temps plein, tels qu'ils apparaissent dans le Mémo Social 1997, sont appliqués à l'ensemble de la population. Cette procédure entraîne une erreur sur les fonctionnaires

3. Seuls les prélèvements obligatoires sont pris en compte : sont exclues, en particulier, les cotisations volontaires des salariés à des régimes de retraite complémentaires. 
qui n'a guère de conséquence sur l'analyse des incitations financières au travail. Elle néglige également les allégements de cotisations patronales sur les embauches nouvelles à temps partiel, pour une durée hebdomadaire de travail comprise entre 18 et 32 heures. Cet allégement réduit de $30 \%$ une partie des cotisations patronales, soit 9,1\% de salaire brut. Mais pour des raisons encore mal élucidées, seuls 400000 emplois bénéficient de la mesure, qui coûte environ 2,5 milliards de francs, soit seulement environ $35 \%$ du total des contrats à durée indéterminée prévoyant un temps partiel entre 16 et 32 heures hebdomadaires. Mention particulière doit être faite des mesures d'allégement des charges sur les bas salaires, qui concernent, en 1997, les salaires inférieurs à 1,33 Smic : la ristourne se traduit par un taux marginal de cotisation sur le coût du travail de $60 \%$ pour les salaires compris entre le Smic et 1,33 Smic (cf. graphique I). Un des résultats annexes de ce calcul est de donner les montants de la CSG non déductible et de la CRDS, qui sont repris dans le calcul de l'impôt sur le revenu.

On a choisi de ne compter que les prestations sociales courantes en espèces dans les transferts, ce qui est évidemment discutable. Dans le cas de la santé, le droit aux soins est général, indépendant du niveau de revenu. La situation est plus complexe en ce qui concerne les retraites. Les droits à retraite sont étroitement dépendants des salaires perçus et des cotisations vieillesse versées : si l'on disposait d'une évaluation de la valeur des droits acquis en fonction du revenu, il conviendrait de l'ajouter aux ressources du ménage. On pourrait également arguer que les cotisations chômage entretiennent un lien actuariel avec les allocations chômage perçues, encore que le risque chômage soit très variable suivant les individus et n'influence pas les cotisations versées. Ces questions, et la façon dont les ménages perçoivent les cotisations sociales comme une épargne génératrice de revenus différés, mériteraient d'être étudiées plus en détail.

\section{L'impôt sur le revenu des personnes physiques : application du barème de 1997 aux revenus salariaux}

L'enquête Emploi ne donne aucune indication sur les revenus non salariaux, et notamment les revenus financiers. Ici, ils sont donc supposés nuls, ce qui, compte tenu de la très forte concentration des revenus financiers, prête surtout à conséquence pour les revenus élevés. Les revenus salariaux des enfants ou des autres personnes partageant le même logement que le chef du ménage et son conjoint sont supposés aussi faire l'objet d'une déclaration fiscale séparée (4). En revanche, on a supposé que tous les couples faisaient une déclaration jointe, alors que les concubins sont tenus de remplir des déclarations séparées. Le barème d'imposition pour les revenus de l'année 1997 est appliqué aux revenus salariaux (salaires nets plus CSG non déductible plus CRDS) en négligeant les déductions diverses non simulées faute d'information (à l'exception des déductions forfaitaires pour enfants scolarisés). Rappelons que les prestations familiales, les allocations logement et le RMI ne sont pas imposables.

\section{Les prestations familiales : des conditions d'attribution complexes}

Les conditions d'attribution des diverses prestations familiales sont complexes et souvent malaisées à déterminer précisément, compte tenu des informations recueillies à l'enquête. La documentation de base utilisée ici est le volume statistique de la Caisse nationale d'allocations familiales (CNAF, 1996), qui retrace la situation au 31 décembre 1996. Sont évaluées les allocations familiales, le complément familial, l'allocation pour jeune enfant, l'allocation parentale d'éducation, l'allocation de parent isolé, l'allocation de rentrée scolaire et les aides à la scolarité.

La plupart des prestations familiales sont définies en référence à la base mensuelle des allocations familiales (BMAF), qui valait 2 108,5 francs en 1997. Ainsi, les allocations familiales sont versées aux familles comprenant au moins deux enfants, sans conditions de ressources en 1997. Elles correspondent à $32 \%$ de la BMAF pour deux enfants et $41 \%$ par enfant supplémentaire, avec des majorations pour les grands enfants. Le complément familial, lui, est de $42 \%$ de la BMAF. Il est versé aux familles comprenant au moins trois enfants de plus de trois ans si les ressources imposables du ménage après abattement ne dépassent pas un plafond de l'ordre de 14000 francs par mois. L'allocation pour jeune enfant (APJE) est d'un montant légèrement supérieur au complément familial et est versée, sous un plafond de ressources identique, aux ménages élevant un enfant de moins de trois ans (5). L'allocation pour parent isolé (API) est

4. Il serait préférable de calculer les revenus du foyer selon que les déclarations sont séparées ou non, en tenant compte des droits à prestations sociales qui peuvent varier selon les cas, et de retenir la formule la plus avantageuse pour le ménage, sous réserve qu'elle ne suscite pas trop de problèmes de répartition. Ceci devrait faire l'objet de travaux complémentaires.

5. Seule l'APJE de troisième période, versée après la naissance de l'enfant, est simulée ici. 
versée aux familles monoparentales élevant au moins un enfant de moins de trois ans. Comme le RMI, c'est une allocation différentielle qui garantit un revenu égal à $200 \%$ ou à $250 \%$ de la BMAF suivant le nombre d'enfants éligibles. Le revenu garanti par l'API est donc supérieur au RMI, mais l'allocation cesse quand les enfants atteignent l'âge de trois ans. L'allocation de rentrée scolaire (ARS), et surtout les aides à la scolarité, sont d'un montant beaucoup plus modeste. Elles sont néanmoins simulées car elles sont soumises à condition de ressources.

L'allocation parentale d'éducation (APE) pose un problème spécifique. Elle est d'environ 3000 francs par mois et est versée, sans conditions de ressources, à tous les ménages ayant au moins deux enfants dont un des parents abandonne son emploi jusqu'à ce que l'enfant concerné atteigne ses trois ans. Faute d'information sur l'activité passée, l'allocation est ici attribuée à tous les couples ayant au moins deux enfants à charge, dont un de moins de trois ans, et dont un membre n'a pas de salaire, sans tenir compte de la condition d'activité antérieure. L'APE réduite, pour les personnes qui exercent une activité à temps partiel (6), n'est pas prise en compte ici, sauf dans les simulations des incitations financières à la reprise d'emploi (cf. infra). Environ $20 \%$ des bénéficiaires de l'APE reçoivent une allocation à taux partiel.

Le cumul des prestations est aussi soumis à certaines conditions. Ainsi, l'APE n'est pas cumulable avec le complément familial ou l'APJE. Le montant de l'APE est supérieur à celui de l'APJE, lui-même supérieur à celui du complément familial ; l'allocation la plus avantageuse pour le ménage parmi celles auxquelles il a droit a été retenue.

Tableau 2

\section{Les prestations familiales}

\section{A - Nombre de ménages bénéficiaires}

\begin{tabular}{|l|c|c|c|}
\hline \multirow{2}{*}{} & \multicolumn{2}{|c|}{ Simulé } & \\
\cline { 2 - 4 } & Population totale & Échantillon restreint & Observé \\
\hline Allocations familiales & 4,4 & 3,7 & 4,5 \\
Complément familial & 1,0 & 0,9 & 0,9 \\
APJE longue & 0,7 & 0,6 & 1,1 \\
APE & 0,6 & 0,5 & 0,5 \\
Allocation rentrée scolaire & 4,0 & 3,1 & 2,9 \\
Bourse scolarité & 1,1 & 0,8 & 0,7 \\
Au moins une prestation & 5,9 & 4,9 & 5,8 \\
\hline
\end{tabular}

B - Montant annuel moyen des allocations

\begin{tabular}{|l|c|c|c|}
\hline \multirow{2}{*}{} & \multicolumn{2}{|c|}{ Simulé } & \multirow{2}{*}{ Observé } \\
\cline { 2 - 4 } \multicolumn{1}{c|}{} & Population totale & Échantillon restreint & \\
\hline Allocations familiales & 16,4 & 16,5 & 15,3 \\
Complément familial & 10,4 & 10,4 & 10,5 \\
APJE longue & 11,2 & 11,2 & 12,8 \\
APE & 36,1 & 36,1 & 28,1 \\
Allocation rentrée scolaire & 1,7 & 1,7 & 1,9 \\
Aides à la scolarité & 1,1 & 1,0 & 1,0 \\
Toutes prestations & 19,9 & 20,6 & - \\
\hline
\end{tabular}

\section{C - Total des prestations versées}

6. Environ 2000 francs par mois pour une durée de travail inférieure au mi-temps et 1500 francs pour une durée comprise entre $50 \%$ et $80 \%$ de la durée légale.

\begin{tabular}{|l|c|r|r|}
\hline \multirow{2}{*}{} & \multicolumn{2}{|c|}{ Simulé } & \\
\cline { 2 - 4 } \multicolumn{1}{c|}{} & \multicolumn{2}{|c|}{ On milliards de francs } \\
\hline Allocations familiales & Population totale & Échantillon restreint & \\
Complément familial & 71,4 & 60,1 & 68,7 \\
APJE longue & 10,7 & 9,0 & 9,7 \\
APE & 7,5 & 6,7 & 13,7 \\
Allocation rentrée scolaire & 20,3 & 18,4 & 12,8 \\
Aides à la scolarité & 6,7 & 5,4 & 5,4 \\
Toutes prestations & 1,1 & 0,8 & 0,7 \\
\hline
\end{tabular}


L'application du module de calcul surestime les allocations versées sous conditions de ressources chaque fois que certains revenus sont absents de l'enquête. Néanmoins, à titre de contrôle de vraisemblance, une estimation a été réalisée sur l'ensemble de la population (ce qui doit donc fournir un chiffre supérieur aux statistiques de la CNAF), à côté du calcul sur la population restreinte dont les revenus sont connus avec plus de précision, mais qui évidemment n'est qu'un sous-ensemble du champ couvert par la CNAF.

La confrontation des résultats de la simulation et des données observées est satisfaisante, à deux exceptions près (cf. tableau 2) : la simulation donne un nombre de bénéficiaires de l'allocation pour jeune enfant trop faible et un montant moyen de l'allocation parentale d'éducation trop élevé. Pour l'APJE, les chiffres du tableau ne concernent que l'APJE dite de troisième période (ou APJE longue), et laissent de côté les APJE versées entre le cinquième mois de la grossesse et le troisième mois de l'enfant, que nous ne savons pas simuler. Mais même sur l'APJE longue, le nombre de bénéficiaires théoriques est nettement inférieur au nombre observé (700 000 ménages au lieu de 1 million). La différence s'explique, en partie, par le fait que cette prestation a été placée sous condition de ressources le 1er janvier 1996, et qu'une fraction des allocataires recensés par la CNAF au 31 décembre 1996 (de l'ordre des deux tiers) bénéficient de versements au titre du régime antérieur, donc pour certains avec des ressources supérieures au plafond d'attribution courant. Pour l'allocation parentale d'éducation, les effectifs comme la prestation moyenne versée sont surestimés. L'écart sur les effectifs n'est pas surprenant : seules les conditions de ressources, de composition familiale et d'inactivité présente sont prises en compte, comme si toutes les personnes qui satisfont ces conditions vérifiaient aussi celle sur l'activité professionnelle antérieure ouvrant un droit à l' APE à taux plein. La différence sur le montant moyen de la prestation s'explique, en partie, par le fait que toutes les APE sont supposées versées à taux plein (environ $20 \%$ des APE sont versées à taux réduit).

Pour les familles monoparentales, la simulation fournit un nombre de bénéficiaires de l'allocation de parent isolé (50 000 ménages) très inférieur au nombre réel (près de 150 000). Le parent isolé est défini ici comme un chef de ménage avec enfants à charge, alors qu'il est vraisemblable qu'un certain nombre de bénéficiaires de l'API vivent chez les grands parents, et par construction nous échappent. Une erreur d'échantillonnage, importante sur de petits effectifs, n'est pas non plus à exclure. En l'absence d'informations, l'autre allocation destinée aux familles monoparentales, l'allocation de soutien familial, destinée aux enfants privés de la pension alimentaire d'un au moins de leurs parents, n'est pas modélisée.

\section{Les allocations logement : un rôle important dans la progressivité des transferts}

Les ménages peuvent bénéficier d'une aide au logement qui dépend de leur revenu imposable, du nombre de personnes du ménage et du type de logement occupé. L'aide personnalisée au logement (APL) concerne les ménages habitant une HLM, tandis que l'allocation logement (AL) s'applique à ceux qui habitent dans le secteur privé. Ces aides au logement ont un rôle très important dans la progressivité du système de transferts : ainsi, un couple sans enfant RMIste habitant une HLM peut percevoir une APL de 1700 francs par mois, tandis que l'APL s'annule si ses revenus salariaux dépassent un seuil proche de 7500 francs par mois. Les aides au logement sont perçues en sus du RMI et les éventuels revenus salariaux des RMIstes ne sont pas pris en compte dans le calcul des allocations logement. Cette dernière disposition évite que le taux marginal dépasse $100 \%$ tant que le ménage touche le RMI, du fait d'une réduction des allocations logement associée à l'accroissement du revenu d'activité. Mais elle a une contrepartie : elle induit une discontinuité dans les revenus nets et un taux marginal de prélèvement infini à la sortie du RMI.

L'application des barèmes pour l'allocation logement et l'aide personnalisée au logement ne va pas de soi : elle suppose connues les charges de loyer ou de remboursement d'emprunt des demandeurs, données absentes de l'enquête Emploi. La simulation taille dans le vif : le loyer de référence des logements situés en zone II (moyenne urbanisation) est attribué à tous les ménages, compte tenu de leur situation familiale. Les ordres de grandeur obtenus sont satisfaisants pour l'APL location, qui concerne les locataires d'une HLM, dans la mesure où peu des ménages écartés précédemment sont susceptibles de bénéficier de l'allocation : 1,8 million de ménages (resp. 30,3 milliards de francs versés) dans la simulation pour 2,2 millions recensés (resp. 36,4 milliards de francs versés) par la CNAF en France métropolitaine. Le barème de l'AL est appliqué à l'ensemble des autres ménages non propriétaires, ce qui recouvre à tort les propriétaires accédants en HLM (650 000 ménages bénéficiaires selon la CNAF) : l'imputation donne 4,1 millions de ménages bénéficiaires (54,2 milliards de francs), alors que les statistiques de la CNAF donnent 3,2 millions (34,8 milliards) pour l'AL. 
Au total, la simulation donne un nombre de bénéficiaires des allocations logement (5,9 millions) très proche de celui recensé dans les statistiques (6,0 millions), mais une dépense totale (84,5 milliards de francs contre environ 73 selon Saunier (1999, p.88-89)) surestimée d'environ $15 \%$.

\section{Une surestimation du nombre simulé de RMIstes}

Le RMI est une allocation différentielle qui garantit à ses bénéficiaires un revenu (non imposable) fonction du nombre de personnes du ménage. Il engendre donc, par définition, un taux marginal de prélèvement de $100 \%$ tant que le ménage reste RMIste. Même sur le champ restreint de la simulation, le calcul des minima sociaux est sujet à erreur. Les conditions d'attribution de ces minima sont très difficiles à reproduire à partir des données de l'enquête (en particulier, le ménage au sens de l'Insee ne coïncide ni avec le foyer fiscal ni avec la notion retenue par la CNAF dans ses statistiques sur les bénéficiaires du RMI (Lhommeau, 1999)). Par ailleurs, on sait que l'estimation de la valeur théorique des allocations chômage, qui dépend de l'activité passée et présente, est quasiment impossible (cf. encadré 1). L'absence d'indications sur les pensions alimentaires conduit aussi à

\section{Encadré 2}

\section{LA DIFFICULTÉ DE REPÉRER LES BÉNÉFICIAIRES DU RMI DANS L'ENQUÊTE EMPLOI}

Dans l'enquête Emploi, on demande aux individus s'ils perçoivent le revenu minimum d'insertion : sur l'ensemble de l'enquête, 540000 personnes en sont bénéficiaires, soit $60 \%$ des effectifs observés par la CNAF. Plusieurs explications (Lhommeau, 1999) ont été avancées pour cette sous-estimation. La question n'est posée qu'aux personnes au chômage, ou cherchant un emploi, ou souhaitant simplement travailler. II se peut que certains bénéficiaires d'une allocation différentielle, exerçant par ailleurs une activité, n'indiquent pas qu'ils perçoivent le RMI.

De plus, l'enquête Emploi n'interroge que les ménages ordinaires, et ne couvre pas les personnes en foyer de travailleurs. En fait, les conditions d'obtention du RMI ne sont pas appliquées aux ménages tels qu'ils sont repérés par les enquêtes de l'Insee : ainsi, sur les 540000 individus mentionnés ci-dessus, seuls 350000 sont la personne de référence du ménage, 50000 le conjoint de la personne de référence, et le reste des enfants ou personnes hébergées dans le même logement. La simulation, qui s'applique au chef du ménage ou à son conjoint, ne prend pas en compte les autres personnes rattachées au ménage, ce qui aurait dû conduire à sous-estimer le nombre de RMlstes. sous-estimer les ressources avant RMI de nombre de femmes isolées.

Toutes ces raisons contribuent à une surestimation très marquée du nombre des RMIstes dans la simulation. Un calcul direct sur l'échantillon restreint donne 2,7 millions de ménages, au sens de l'enquête, susceptibles de bénéficier du RMI. Si on se limite aux ménages dont le chef (et éventuellement son conjoint) a 55 ans au plus (7), il reste 2,2 millions de ménages bénéficiaires potentiels, alors que les statistiques dénombrent moins de 1 million de RMI distribués fin 1996. Dans l'enquête, de nombreux ménages sont sans ressources apparentes et le programme d'imputation leur assigne mécaniquement le RMI. L'enquête Emploi donne un montant d'allocations chômage qui n'a pas été retenu dans les calculs (8). Lorsqu' on l'incorpore, l'imputation ramène le nombre de ménages RMIstes de 2,2 à 1,8 million, ce qui reste très supérieur à la réalité. Il est vraisemblable qu'à la fois une information plus fiable sur les allocations chômage, la connaissance des versements au titre de l'allocation aux adultes handicapés et celle des pensions alimentaires perçues rapprocheraient les estimations des chiffres enregistrés. Le 31 décembre 1996, 513000 chômeurs recevaient l'allocation de solidarité spécifique (Saunier, 1999, p. 56). L'allocation pour adulte handicapé (AAH) concernait 344000 bénéficiaires à taux plein et 226000 à taux réduit à la même date. C'est une allocation différentielle, comme le RMI, mais dont le plafond est celui du minimum vieillesse et dépasse donc celui du RMI d'à peu près un tiers : environ 3400 francs par mois pour un bénéficiaire seul à taux plein. Quant aux pensions alimentaires, selon des données tirées de l'enquête Budget de Famille de 1995, environ 500000 ménages en reçoivent, pour 12 milliards de francs au total, soit 2000 francs par mois et par ménage bénéficiaire (9). Elles représentent environ $15 \%$ des ressources des ménages bénéficiaires (un quart pour les personnes seules) et constituent donc un apport de revenus non négligeable qui peut faire sortir nombre de ménages du RMI. Il n'est pas impossible par ailleurs qu'il y ait des personnes qui aient droit au RMI mais n'en fassent pas la demande (cf. encadré 2). Au total, l'essentiel de la

\footnotetext{
7. Nous n'avons pas d'information sur les préretraites.

8. Le total des allocations chômage versées selon l'enquête s'élève à 95 milliards de francs, contre 127 milliards en 1997 selon les statistiques macro-économiques. Nous n'avons pas utilisé les chiffres d'allocations chômage de l'enquête pour les calculs d'incitation rapportés ci-après. Ceci conduit à attribuer le RMI à des ménages qui ont en fait des allocations chômage supérieures au $R M I$, et probablement à sous-estimer l'effet désincitatif du système de protection sociale.

9. Les auteurs remercient Anne Flipo, qui leur a fourni ces évaluations.
} 
surestimation du nombre de RMIstes semble provenir d'un mauvais classement des adultes handicapés titulaires de l'AAH et des chômeurs recevant l'allocation de solidarité spécifique.

\section{Les RMIstes sont exemptés du paiement de la taxe d'habitation}

Comme on l'a vu plus haut, on se limite ici a priori aux prélèvements et transferts en espèces liés aux revenus du travail, en excluant les autres composantes du comportement des ménages (éducation, services de santé, demande de biens de consommation, etc.). Aussi, la taxe à la valeur ajoutée est-elle hors champ, et on pourrait également laisser de côté la taxe d'habitation, assise sur la valeur locative des logements. Mais cette taxe a un aspect redistributif lié au revenu. Au-delà des variations commune par commune, la taxe d'habitation est en effet sujette à des exonérations totales ou partielles nationales : les RMIstes ne payent pas la taxe d'habitation et les foyers fiscaux de revenu imposable peu élevé bénéficient de réductions. Ces déductions sont retracées au bas de l'échelle des revenus. L'enquête ne fournit pas, bien sûr, la valeur locative cadastrale des logements, ni les caractéristiques locales de l'impôt. Le loyer forfaitaire retenu est le même que celui de l'allocation logement. De manière un peu arbitraire, on lui applique le taux moyen de $10 \%$ (10), duquel on retranche les exonérations nationales. Ce mode de calcul, assez grossier, néglige nombre de sources de variations locales (valeurs locatives, taux de la taxe, exonérations facultatives). Il a néanmoins le mérite de modéliser la variation de la taxe avec le revenu, et en particulier le taux marginal de prélèvement infini induit par la taxe d'habitation à la sortie du RMI (qui vient s'ajouter à la chute brutale des allocations logement déjà signalée).

\section{Les incitations financières au travail}

T e programme d'imputation permet de procéder Là trois exercices pour décrire, de divers points de vue, la façon dont le système socio-fiscal affecte les incitations au travail. On se limite ici aux incitations purement financières. On évalue le taux marginal de prélèvement, c'est-à-dire la somme prélevée par le système socio-fiscal quand l'employeur augmente de 100 francs le coût du travail de l'individu considéré (le complémentaire venant accroître le revenu disponible du ménage). Dans le même esprit, on estime ensuite l'augmentation de coût salarial nécessaire, du côté de l'employeur, pour que le ménage dont fait partie l'individu concerné obtienne in fine 1000 francs de plus par mois en net.

Les deux exercices précédents se fixent des paramètres (100 et 1000 francs) nécessairement un peu arbitraires. Par ailleurs, ils n'ont qu'une signification limitée pour les chômeurs et les inactifs : ils correspondraient, dans leur cas, à un emploi à temps partiel (voire à quelques heures de travail par mois). Pour ces deux catégories, il est utile de compléter l'analyse par l'étude des effets du système socio-fiscal sur les incitations financières à reprendre un emploi. Ceci pose inévitablement la question de la rémunération associée à un tel emploi, qui doit normalement dépendre des caractéristiques de l'individu concerné. À titre illustratif, dans un troisième exercice, on estime l'augmentation de ressources nettes des ménages dans l'éventualité où un de ses membres chômeur ou inactif trouverait un emploi à plein temps ou à mi-temps rémunéré au Smic.

\section{Les taux de prélèvement marginaux sont les plus élevés pour les revenus les plus bas}

Chaque personne de l'échantillon restreint, représentant 19,9 millions d'individus, est supposée recevoir une augmentation de salaire correspondant à un accroissement marginal de coût du travail pour l'employeur (potentiel) de 100 francs. Une partie de ces 100 francs sert à acquitter les cotisations sociales, la CSG et la CRDS ; le reste vient accroître les revenus salariaux (éventuellement nuls au départ) de l'individu concerné. L'augmentation du revenu imposable qui en résulte peut avoir pour conséquence d'accroître l'impôt sur le revenu acquitté par le ménage auquel il appartient, ou de réduire les prestations familiales et les allocations logement que ce ménage peut percevoir. Pour les RMIstes qui restent au RMI, l'augmentation des revenus salariaux est entièrement compensée par une baisse du RMI perçu. Pour les autres ménages, le revenu disponible augmente en général. $\mathrm{Si}$ $R(w, C)$ désigne la ressource mensuelle nette du ménage après impôts et transferts sociaux, où $w$ désigne le coût du travail de la personne considérée et $C$ toutes les caractéristiques du ménage qui rentrent dans la détermination des ressources (salaire du conjoint, nombre et âge des enfants, nature du logement, etc.), la ressource mensuelle nette passe

10. La taxe d'habitation a rapporté 70,7 milliards de francs après exonérations en 1997, et l'ensemble des loyers, y compris les loyers fictifs des propriétaires occupants, est de 793 milliards selon la comptabilité nationale de cette même année. 
à $R(w+100, C)$. Le taux de prélèvement marginal est alors défini (en pourcentage) par $100-(R(w+100, C)-R(w, C))$.

Ce calcul reproduit sur les données de l'enquête Emploi les résultats théoriques associant les barèmes à des cas-types de la direction de la Prévision

\section{Graphique II}

Taux de prélèvements marginaux

A - Fonction de répartition des taux marginaux

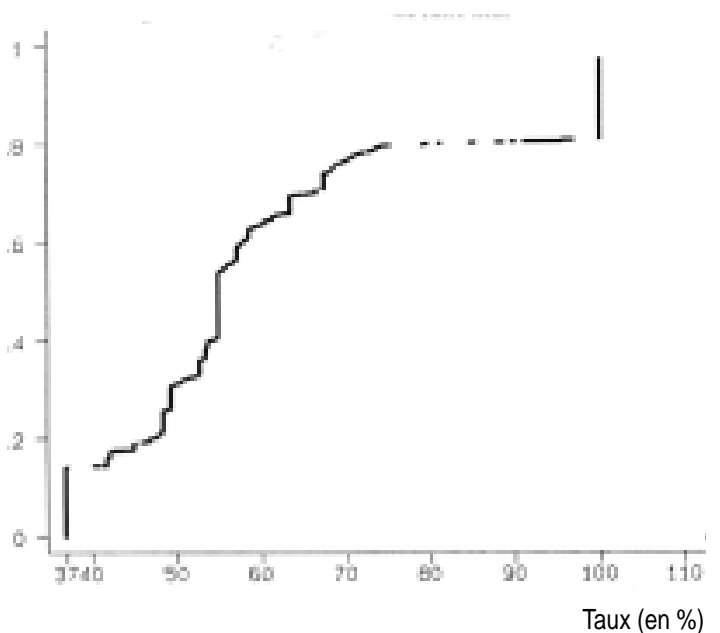

B - Distribution des taux marginaux selon les salaires nets reçus par le ménage

Taux marginal (en \%)

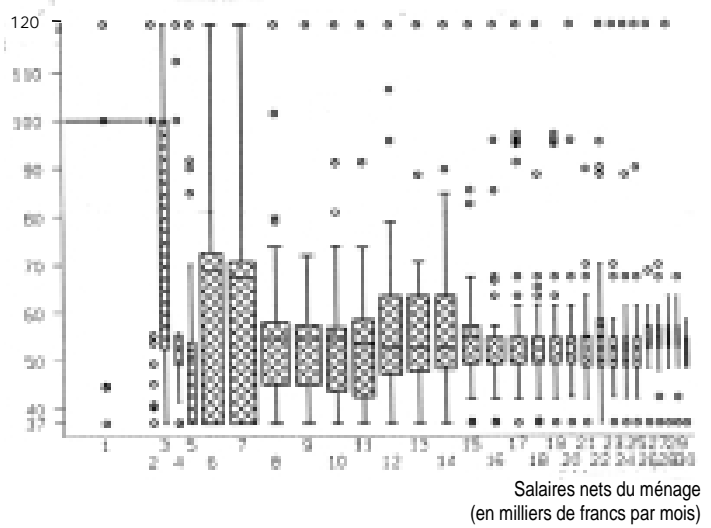

Lecture : cette représentation graphique est due à Tukey. Pour chaque tranche, la boîte, dont la largeur est proportionnelle à la taille de la population concernée, s'étend du premier quartile au troisième quartile des taux marginaux, le trait horizontal dans la boîte représentant le taux médian. Les traits verticaux qui s'étendent de chaque côté de la boîte fournissent des indications sur les queues de distribution. On calcule une distance de référence, égale à 1,5 fois l'intervalle interquartile (la hauteur de la boîte). Les traits s'étendent du quartile (premier ou troisième) jusqu'à l'observation la plus éloignée dans la limite de la distance de référence. Les ronds enfin représentent toutes les observations individuelles qui tombent au-delà de la plage délimitée par ces traits. Le graphique correspond à un tirage aléatoire au cinquième de la population.

Source : calculs des auteurs.
(Paillaud et Eyssartier, 1998), ce qui permet d'étudier la répartition des taux marginaux de prélèvement dans la population française. Le tableau 3 et le graphique II-A présentent la distribution de ces taux marginaux dans la population étudiée. Le tableau 3 donne la répartition de la population selon les valeurs du taux par tranche. La plus basse tranche de prélèvement, $35-45 \%$ et $19,4 \%$ de la population, comprend essentiellement des personnes recevant un salaire bénéficiant de la ristourne maximale de cotisations employeurs sur les bas salaires, donc inférieur au SMIC (cotisations égales à $37 \%$ du coût du travail), et pour qui l'augmentation ne se traduit ni par une réduction des prestations soumises à conditions de ressources ni par une augmentation de l'IRPP, que ces ménages n'acquittent généralement pas. Les tranches suivantes, entre 45 et $65 \%$, regroupent la moitié de la population. Elles correspondent souvent à des salaires assez élevés, au-dessus de 1,33 Smic : les salariés concernés supportent des taux de cotisations sociales non affectés par la ristourne bas salaires et peuvent être assujettis à l'IRPP. Au-dessus de $60 \%$, on trouve notamment, aux côtés des ménages riches dans les hautes tranches de l'impôt sur le revenu, les salariés recevant un salaire compris entre le Smic et 1,33 Smic : le taux marginal de cotisation qui raccorde les allégements sur bas salaires au taux normal au-delà de 1,33 Smic est en effet déjà de $60 \%$. Enfin, près de $20 \%$ de la population sont confrontés à un taux marginal supérieur à $90 \%$. On trouve là pour l'essentiel tous les membres des ménages bénéficiaires d'allocations différentielles, revenu minimum d'insertion ou allocation de parent isolé, juste au-delà des plafonds d'attribution du complément familial ou de l'allocation pour jeune enfant, mais aussi des personnes qui se trouvent localement à des points de discontinuité, associés à la perte de certaines allocations (allocations logement) ou à des seuils minima d'imposition (IRPP).

Tableau 3

Taux de prélèvement pour une variation marginale de coût du travail de $\mathbf{1 0 0}$ francs (distribution dans la population étudiée)

\begin{tabular}{|l|c|c|}
\hline Taux en \% & Nombre (millions) & Pourcentage \\
\hline $35-45$ & 3,9 & 19,4 \\
$45-55$ & 7,0 & 35,3 \\
$55-65$ & 3,0 & 15,1 \\
$65-75$ & 2,0 & 10,0 \\
$75-85$ & 0,1 & 0,6 \\
$85-95$ & 0,1 & 0,3 \\
$>95$ & 3,8 & 19,3 \\
\hline Total & 19,9 & 100,0 \\
\hline
\end{tabular}

Source : calculs des auteurs. 
Le graphique II apporte un éclairage complémentaire. Le graphique II-A présente la fonction de répartition des taux marginaux dans la population : pour chaque valeur $x$ du taux en abscisse, on représente la proportion des individus dans la population qui sont confrontés à un taux marginal inférieur ou égal à $x$ (du fait des discontinuités, il y a quelques taux très élevés, supérieurs à $120 \%$ : pour la lisibilité du graphique, on a ramené tous ces taux à 100). On observe quelques points de concentration, $37 \%$, $55 \%$ et $100 \%$, associés à plus de $15 \%$ de la population. On a décrit ci-dessus les sous-populations confrontées aux taux de $37 \%$, les bas salaires, et de $100 \%$, les RMIstes. Le taux de $55 \%$ correspond pour l'essentiel à des salariés qui perçoivent un salaire compris entre 1,33 Smic et le plafond de la Sécurité sociale, dont le taux de cotisations sociales, CRDS et CSG, calculé sur le coût du travail, est de $45 \%$, et qui, de surcroît, se trouvent dans la deuxième tranche de l'IRPP, avec un revenu imposable par part compris entre 50990 et 89650 francs.

Le graphique II-B donne quelques caractéristiques de la distribution des taux marginaux par tranches de salaires nets du ménage. On s'est limité aux ménages dont les salaires nets sont inférieurs à 30000 francs par mois, par tranche de 1000 francs : la tranche 1 correspond aux ménages de salaires nets compris entre 0 et 1000 francs, et ainsi de suite. Le taux de $100 \%$ est supporté essentiellement par les ménages à bas revenus salariaux (moins de 3000 francs par mois). Pour les deux premières tranches, premier et troisième quartiles sont égaux à 100, de sorte que la «boîte » est réduite à un segment : la quasi-totalité de la population reçoit des allocations différentielles (RMI, allocation de parent isolé, etc.) et se voit confisquer à terme un accroissement marginal de salaire (11). Au-delà des deux premières tranches, on est frappé par la grande dispersion des taux marginaux des individus à l'intérieur d'une même tranche de salaires nets de leur ménage, qui tient à la composition de ces salaires (un ou deux salaires), au nombre et à l'âge des enfants, à la nature du logement occupé, etc. Si l'on met à part les personnes imposées à $37 \%$ qui, comme on l'a vu plus haut, pour l'essentiel se trouvent dans des ménages dont les salaires nets sont compris entre 4000 et 7000 francs, les taux semblent baisser avec les salaires nets dans la plage inférieure à 12000 francs mensuels. Les valeurs observées sont élevées, du fait des cotisations sociales entre 1 et 1,33 Smic et de la dégressivité des aides au logement. Au-dessus de 12000 francs par mois, on décèle une remontée lente, à peine perceptible, qui est peut être minorée par la non-prise en compte des revenus du capital dans la simulation.
Cet examen rapide confirme un des enseignements des études de cas. D'une part, la progressivité du système socio-fiscal pris dans son ensemble paraît limitée, au moins pour les ménages dont les revenus salariaux sont inférieurs à 25000 francs par mois. Notre étude ne permet pas de conclure pour les revenus salariaux plus élevés, dans la mesure où leurs bénéficiaires perçoivent aussi des revenus de capitaux élevés dont nous n'avons pas connaissance et qui peuvent les amener dans des tranches supérieures de l'impôt sur le revenu. D'autre part, ce sont les personnes qui ont les revenus les plus bas qui ont les taux marginaux les plus élevés. C'est une conséquence de l'État Providence : la redistribution opère des transferts au profit des plus pauvres et, comme on doit prélever les ressources correspondantes sur les plus riches, il faut des taux marginaux de prélèvement très élevés au passage entre la pauvreté et l'autosuffisance. La taille de la population concernée et la distribution des taux selon les revenus salariaux sont des résultats nouveaux quelque peu surprenants, qui méritent confirmation par des études indépendantes. Il convient toutefois de rappeler que l'évaluation présentée ici est sensible aux incertitudes qui pèsent sur les bas revenus. Ainsi, une personne isolée sans ressources imposables bénéficie du RMI dans notre calcul et de ce fait est soumise à un taux de prélèvement marginal de $100 \%$. Si elle avait, en fait, droit à des allocations chômage, la reprise d'un emploi induirait un taux marginal inférieur à $100 \%$ pendant une éventuelle période d'intéressement, puis un taux marginal infini dans l'optique de long terme retenue ici. En revanche, un titulaire de l'AAH est bien soumis à un taux marginal de $100 \%$. Les choses sont plus compliquées pour une femme touchant une pension alimentaire, puisque le taux marginal qui s'applique à elle peut dépendre des modalités du divorce.

\section{Le coût salarial d'un accroissement des ressources nettes du ménage de 1000 francs}

Dans ce deuxième exercice, on calcule, pour chaque personne de l'échantillon, l'augmentation de coût salarial (ou le niveau de coût salarial pour celles n'ayant pas à l'origine d'emploi salarié) qui conduit à un accroissement des ressources mensuelles nettes du ménage auquel elle appartient de 1000 francs. En pratique, ceci revient à rechercher

11. Rappelons que nous nous plaçons dans une optique de long terme, et que nous n'avons pas pris en compte les mécanismes d'" intéressement " qui réduisent à court terme le taux marginal (Paillaud et Eyssartier, 1999). 
pour chaque individu la solution en $\Delta w$ de l'équation (cf. encadré 3) :

$R(w+\Delta w, C)=R(w, C)+1000$

Le tableau 4 et le graphique III décrivent quelques caractéristiques des ménages par grandes tranches de $\Delta w$, et les modifications des diverses catégories de prélèvements et transferts au cours de la transformation considérée. Pour plus de $80 \%$ de la population étudiée, la hausse de coût du travail nécessaire est comprise entre 1500 et 3500 francs. On trouve ici la plus grande partie des salariés, qui appartiennent à des ménages dont les revenus salariaux nets sont supérieurs à 7000 francs par mois. Les ménages concernés subissent typiquement un faible accroissement d'impôt sur le revenu et peu de modifications sur les prestations perçues, hors l'allocation logement qui joue significativement jusqu'à 12000 francs de salaires nets mensuels. À l'intérieur de cette catégorie, la différence la plus importante vient du passage du coût du travail au salaire net, autrement dit des cotisations sociales : les salariés payés au-dessus de 1,33 Smic supportent des taux marginaux de cotisations sociales entre 35 et $40 \%$ du coût du travail, alors que ce taux est proche de $60 \%$ pour ceux dont le salaire est compris entre le Smic et 1,33 Smic, qui bénéficient de la ristourne sur les bas salaires. La hausse de coût du travail des premiers est inférieure à 2500 francs, celle des seconds est souvent comprise entre 2500 et 3500 francs.

La tranche suivante, variation du coût du travail entre 3500 et 4500 francs, correspond à des ménages dont les salaires nets sont, en moyenne, beaucoup plus faibles : 6600 francs par mois contre à

Tableau 4

Hausse des ressources du ménage de 1000 francs par mois

Accroissement du coût du travail conduisant à une hausse des ressources nettes mensuelles de 1000 francs (distribution de la population par tranches et décomposition)

\begin{tabular}{|c|c|c|c|c|c|c|c|c|}
\hline Tranche & $\begin{array}{l}1500- \\
2500 \\
\end{array}$ & $\begin{array}{r}2500- \\
3500 \\
\end{array}$ & $\begin{array}{l}3500- \\
4500 \\
\end{array}$ & $\begin{array}{l}4500- \\
5500 \\
\end{array}$ & $\begin{array}{l}5500- \\
6500 \\
\end{array}$ & $\begin{array}{l}6500- \\
7500 \\
\end{array}$ & $\begin{array}{l}7500- \\
8500\end{array}$ & $>8500$ \\
\hline $\begin{array}{l}\text { Nombre (millions) } \\
\text { Pourcentage } \\
\text { Salaire net du ménage }\end{array}$ & $\begin{array}{r}13,0 \\
65,6 \\
13010 \\
\end{array}$ & $\begin{array}{r}3,1 \\
15,5 \\
11363 \\
\end{array}$ & $\begin{array}{r}0,5 \\
2,7 \\
6639 \\
\end{array}$ & $\begin{array}{r}0,4 \\
2,1 \\
2708 \\
\end{array}$ & $\begin{array}{r}0,7 \\
3,5 \\
2535 \\
\end{array}$ & $\begin{array}{r}1,0 \\
4,8 \\
491 \\
\end{array}$ & $\begin{array}{r}0,5 \\
2,3 \\
767 \\
\end{array}$ & $\begin{array}{r}0,7 \\
3,5 \\
109 \\
\end{array}$ \\
\hline Coût du travail & 2075 & 2874 & 3947 & 5087 & 6075 & 6697 & 7815 & 9022 \\
\hline $\begin{array}{l}\text { Charges salariales } \\
\text { Salaire net }\end{array}$ & $\begin{array}{r}863 \\
1211 \\
\end{array}$ & $\begin{array}{l}1513 \\
1361 \\
\end{array}$ & $\begin{array}{l}1922 \\
2025 \\
\end{array}$ & $\begin{array}{l}1925 \\
3162 \\
\end{array}$ & $\begin{array}{l}2238 \\
3837 \\
\end{array}$ & $\begin{array}{l}2465 \\
4232 \\
\end{array}$ & $\begin{array}{l}2894 \\
4921 \\
\end{array}$ & $\begin{array}{l}3567 \\
5455\end{array}$ \\
\hline $\begin{array}{l}\text { - IRPP } \\
\text { Prestations familiales } \\
\text { Allocation parent isolé } \\
\text { RMI } \\
\text { Allocation logement } \\
\text { - Taxe d'habitation }\end{array}$ & $\begin{array}{r}-145 \\
-4 \\
0 \\
-1 \\
-62 \\
0\end{array}$ & $\begin{array}{r}-134 \\
-21 \\
0 \\
-58 \\
-136 \\
-13\end{array}$ & $\begin{array}{r}-224 \\
-175 \\
-29 \\
-248 \\
-285 \\
-39\end{array}$ & $\begin{array}{r}-41 \\
-366 \\
0 \\
-1460 \\
-186 \\
-110\end{array}$ & $\begin{array}{r}-29 \\
-691 \\
-1 \\
-1462 \\
-565 \\
-90\end{array}$ & $\begin{array}{r}-9 \\
-138 \\
-1 \\
-2377 \\
-573 \\
-135\end{array}$ & $\begin{array}{r}-30 \\
-118 \\
-133 \\
-2944 \\
-525 \\
-143\end{array}$ & $\begin{array}{r}-4 \\
-20 \\
0 \\
-3341 \\
-934 \\
-157\end{array}$ \\
\hline
\end{tabular}

Source : calculs des auteurs.

Encadré 3

\section{REMARQUE TECHNIQUE SUR LA DISCONTINUITÉ DE LA FONCTION DE REVENU R}

Plusieurs caractéristiques du système socio-fiscal expliquent que la fonction $\mathrm{R}$ n'est pas continue en $\mathrm{w}$. La plupart des discontinuités sont à la baisse et induisent donc un taux marginal de prélèvement infini. C'est le cas de la plus importante qui est liée à la baisse brutale des allocations logement et à la perception de la taxe d'habitation à la sortie du RMI. Comme la fonction $\mathrm{R}$ redevient croissante après une telle discontinuité, l'équation possède quand même une solution. Or, de façon plus surprenante, la fonction $R$ possède également une discontinuité à la hausse. Celle-ci tient à la modalité de détermination du plafond de ressources imposables annuelles associé au versement du complément familial ou de l'allocation pour jeune enfant. Ce plafond, qui dépend du nombre et de l'âge des enfants, est majoré de 33973 francs par an pour les familles monoparentales et pour les familles dont les deux conjoints travaillent et gagnent chacun un salaire net mensuel supérieur à la base mensuelle des allocations familiales (BMAF). Lorsque le salaire d'un membre d'un couple dont l'autre conjoint gagne plus de la BMAF franchit celle-ci, les ressources du ménage qui satisfait les autres conditions d'attribution du complément familial ou de l'APJE, augmentent subitement de 879 francs (montant du complément familial) ou de 969 francs (montant de l'APJE). Si avant le seuil, on avait $R(w+w, C)<R(w, C)+1000$, compte tenu des montants en jeu, il est vraisemblable qu'au-delà, on aura $R(w+w, C)>R(w, C)+1000$ : l'équation n'a pas de solution. C'est le seul cas rencontré où une augmentation de salaire peut conduire à une augmentation très supérieure des ressources (le taux marginal de prélèvement est en toute rigueur égal à moins l'infini !). Ceci ne concerne heureusement que seulement 37 observations de l'échantillon, représentant 12000 individus, sur un total de 71021 observations. Ils ont donc été écartés du calcul. 
Graphique III

Variation de salaire conduisant à un accroissement de 1000 francs des ressources du ménage

A - Coût du travail

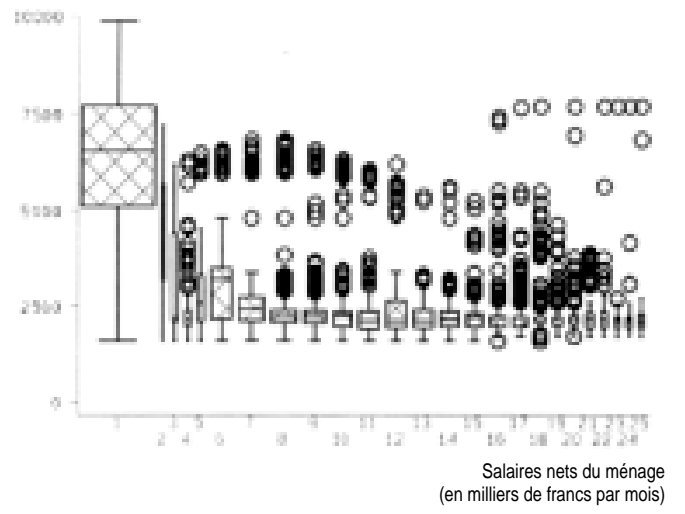

B - Cotisations sociales

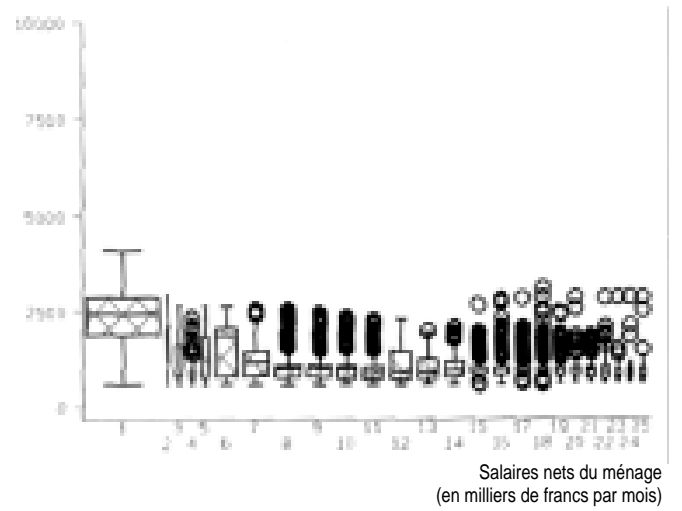

C - Salaire net (égale A-B)

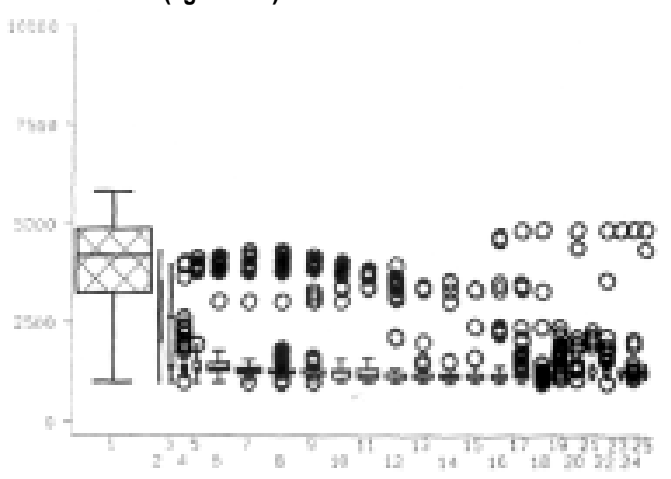

D - RMI

Salaires nets du ménage

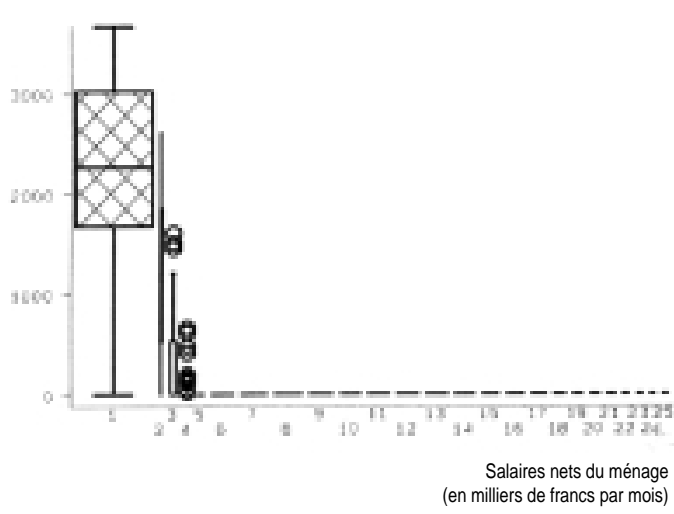

Source : calculs des auteurs.
E - Prestations familiales

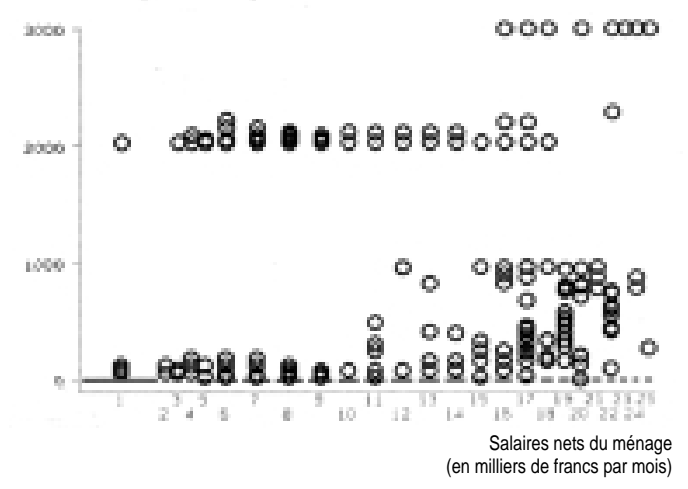

F - Allocations logement

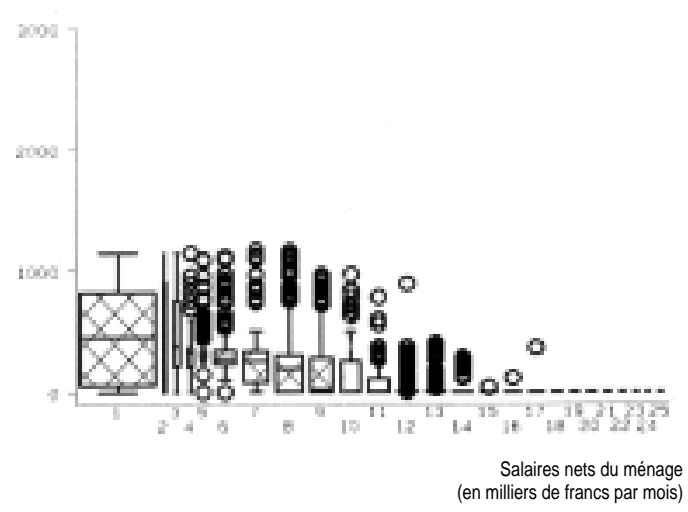

G - IRPP

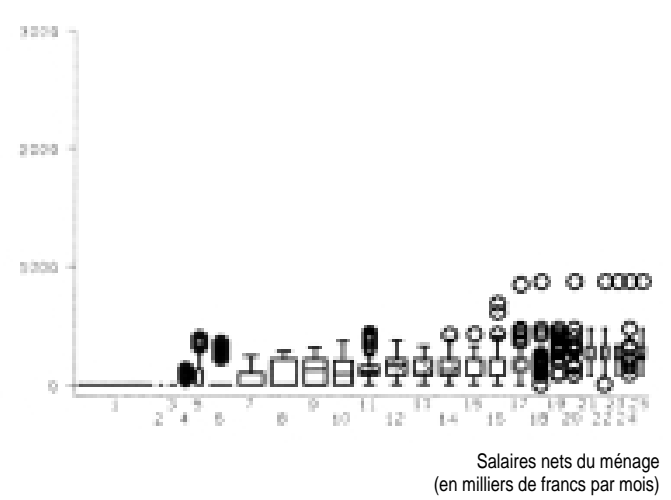

Lecture : ce graphique détaille les variations du coût du travail, et des divers prélèvements et transferts associés, qui conduisent à un accroissement des ressources nettes du ménage de 1000 francs par mois. Ces variations sont décrites en fonction du salaire net initial de la personne considérée, à l'aide de la représentation de Tukey déjà utilisée dans le graphique II. Si on se concentre, par exemple, sur les personnes ayant les salaires nets les plus bas, pour obtenir un accroissement de 1000 francs des ressources nettes de leur ménage, il faut une augmentation médiane de coût de travail de 6500 francs (graphique A), soit 2500 francs de cotisations sociales (graphique B) et 4000 francs de salaire net (graphique C). De ces 4000 francs de salaire net, 2400 sont absorbés par une baisse du RMI (graphique $D$ ) et 600 par une baisse des allocations logement, les prestations familiales perçues et l'impôt sur le revenu payé étant pratiquement inchangés (graphiques $E, F$ et $G$ ). 
peu près 12000 francs précédemment. Beaucoup de ces ménages reçoivent des transferts sociaux sous conditions de ressources, et il faut accroître le salaire net de façon marquée pour compenser la perte de ces transferts associée à la hausse postulée de 1000 francs des ressources mensuelles totales. Cette catégorie de ménages perçoit souvent des prestations familiales sous conditions de ressources (le graphique III-E montre toutefois que les prestations familiales sont perçues par moins d'un quart des ménages, quelle que soit la tranche de salaires étudiée). Les effets des allocations logement sont importants, supérieurs à ceux de l'IRPP. Dans les tranches supérieures, au-dessus de 4500 francs de hausse du coût du travail, les ménages concernés n'ont plus de ressources salariales (14\% environ de la population étudiée est dans ce cas). Ici joue à plein le caractère différentiel du RMI : le salaire doit compenser complètement la perte du RMI avant de permettre un accroissement des ressources du ménage. Les allocations logement sont également fortement réduites lorsque le ménage sort du RMI. L'augmentation de salaire doit être d'autant plus importante que le ménage est plus

\section{Tableau 5}

Occupation et incitation au travail salarié

Accroissement du coût du travail conduisant à une hausse des ressources nettes mensuelles de 1000 francs (décomposition selon l'activité )

\begin{tabular}{|l|c|c|c|c|}
\hline \multicolumn{1}{|c|}{ Tranche } & Actif occupé & Chômeur & Inactif & Ensemble \\
\hline Nombre (millions) & 14,8 & 2,0 & 3,1 & 19,9 \\
Pourcentage & 74,5 & 10,1 & 15,5 & 100,0 \\
Salaire net du ménage & 12684 & 3562 & 5582 & 3051 \\
\hline Coût du travail & 2719 & 4630 & 3619 & 1282 \\
\hline Charges salariales & 1205 & 1735 & 1357 & 1769 \\
Salaire net & 1514 & 2895 & 2263 & -125 \\
\hline - IRPP & -154 & -27 & -50 & -52 \\
Prestations familiales & -17 & -51 & -225 & -4 \\
Allocation parent isolé & 0 & -13 & -17 & -397 \\
RMI & -222 & -1308 & -278 & -165 \\
Allocation logement & -108 & -414 & -39 & -24 \\
- Taxe d'habitation & -14 & -79 & & \\
\hline
\end{tabular}

Source : calculs des auteurs.

Tableau 6

Effet de la reprise d'un emploi (niveau)

Hausse en francs des ressources mensuelles nettes des ménages dont un membre non salarié prend un emploi (distribution en pourcentage parmi les chômeurs et inactifs sans salaire déclaré)

\begin{tabular}{|c|c|c|c|}
\hline \multicolumn{4}{|c|}{ Emploi à temps plein rémunéré au Smic } \\
\hline Gain net en francs & Chômeurs & Inactifs & Ensemble \\
\hline Moins de 1000 & 12,2 & 6,9 & 9,0 \\
\hline $1000-2000$ & 32,5 & 20,4 & 25,3 \\
\hline $2000-3000$ & 8,7 & 8,6 & 8,6 \\
\hline $3000-4000$ & 20,5 & 33,1 & 28,0 \\
\hline Plus de 4000 & 26,2 & 31,1 & 29,1 \\
\hline Total & 100,0 & 100,0 & 100,0 \\
\hline Total (en millions) & 1,9 & 2,9 & 4,9 \\
\hline \multicolumn{4}{|c|}{ Emploi à mi-temps rémunéré au Smic } \\
\hline Gain net en francs & Chômeurs & Inactifs & Ensemble \\
\hline Moins de 500 & 54,0 & 33,9 & 42,0 \\
\hline $500-1000$ & 3,0 & 5,1 & 4,2 \\
\hline $1000-1500$ & 2,0 & 1,8 & 1,9 \\
\hline $1500-2000$ & 20,0 & 18,7 & 19,2 \\
\hline Plus de 2000 & 21,1 & 40,5 & 32,7 \\
\hline Total & 100,0 & 100,0 & 100,0 \\
\hline Total (en millions) & 1,9 & 2,9 & 4,9 \\
\hline
\end{tabular}

Source : calculs des auteurs. 
aidé, par exemple s'il a de fortes charges de famille. Pour $3,8 \%$ de la population, la hausse du coût du travail est supérieure à un Smic à temps plein, soit 7970 francs. Une telle situation peut paraître surprenante, mais s'explique facilement. Un couple au chômage avec un enfant bénéficie d'un RMI d'environ 3500 francs et d'environ 2000 francs d'allocation logement, soit 5500 francs par mois. Si l'un des membres du couple touche un Smic, soit 5000 francs nets, le couple perdra tout son RMI et sera affecté par la chute des allocations logement au sortir du RMI (soit environ 350 francs par mois) et devra payer la taxe d'habitation (à peu près 150 francs). Son revenu disponible deviendra $5000+(2000-350)-150=6500$ francs, soit un accroissement net de ressources de 1000 francs par mois.

Le fait que les individus les moins favorisés sont ceux qui ont le moins d'incitations financières à l'effort au travail se retrouve dans le tableau 5. Ce tableau présente les mêmes statistiques que le tableau 4 pour les actifs occupés, les chômeurs et les inactifs. Un chômeur, pour augmenter les ressources nettes de son ménage de 1000 francs par mois, doit, en moyenne, accroître son salaire net de 2900 francs, dont près de la moitié sert à compenser la perte du RMI, alors qu'il suffit de 1500 francs pour un actif. Pour présenter les choses autrement, un accroissement des ressources nettes mensuelles du ménage de 1000 francs est obtenu à un coût moyen pour l'employeur de 4600 francs si l'individu est chômeur, et de 2700 francs s'il est actif occupé.

\section{Les incitations financières à la reprise d'un emploi}

La dernière simulation s'intéresse au cas des chômeurs et inactifs, qui n'ont pas de ressources salariales. Les deux calculs précédents ne sont alors pas les plus pertinents. Il est plus naturel de regarder l'avantage financier que ces personnes retirent de la prise d'un emploi, à temps plein ou à temps partiel, rémunéré au Smic. La distribution des gains nets après impôts et transferts sociaux de ces 4,9 millions d'individus est décrite dans le tableau 6. Les situations sont très hétérogènes (cf. encadré 4 ). Plus de $60 \%$ des inactifs retirent une somme supérieure à 3000 francs par mois d'un emploi à temps plein, mais moins de la moitié des chômeurs. La différence vient de ce que les inactifs ont plus souvent que les chômeurs un conjoint salarié, et ne bénéficient donc pas du RMI ou de transferts sociaux sous conditions de ressources. Les $40 \%$ de chômeurs (environ 850000 personnes) qui gagnent moins de 2000 francs par mois lorsqu'ils prennent un emploi à temps plein au Smic sont essentiellement des RMIstes.

\section{Encadré 4 \\ LES CONSÉQUENCES FINANCIÈRES D'UNE REPRISE D'EMPLOI SONT DIFFICILES À MESURER}

Les évaluations des tableaux 6 et 7 dépendent fortement de la modélisation retenue des minima sociaux, et tout particulièrement du RMI. La procédure d'imputation retenue ici surestime d'environ 1 million le nombre de RMIstes, probablement en faisant figurer à tort dans cette catégorie 500000 bénéficiaires de l'allocation aux adultes handicapés (AAH) et autant de chômeurs recevant l'allocation de solidarité spécifique (ASS). L'AAH est une allocation différentielle de montant supérieur au $\mathrm{RMI}$, et la possible erreur de classement des handicapés est sans conséquence sur le résultat des deux tableaux précédents. Les modalités d'attribution de l'ASS sont un peu différentes : le ménage reçoit l'allocation à taux plein (environ 2300 francs par mois) tant que ses ressources ne dépassent pas un certain plafond (2 900 francs pour un célibataire) ; au-dessus l'allocation est différentielle. Prendre un emploi à mi-temps rémunéré au Smic n'a donc pas les mêmes conséquences pour un RMIste et un titulaire de l'ASS. Pour le premier, s'il est isolé, cela se traduit par la sortie du RMI et souvent une perte financière à cause des effets pervers de l'attribution de l'allocation logement. Pour le second, en l'absence d'autres ressources, le caractère différentiel de l'ASS joue, mais à un niveau de ressources plus élevé d'environ 5200 francs mensuels. La " trappe à pauvreté " apparaît pour des salaires supérieurs, par exemple dans le cas d'une reprise d'emploi à temps plein, qui doit se traduire dans des termes plus proches pour le RMIste et le titulaire de l'ASS (1). $\mathrm{Ce}$ raisonnement qualitatif laisse à penser que l'essentiel de l'incertitude porte sur les conséquences financières d'une reprise d'emploi à mi-temps. Si effectivement les 500000 chômeurs bénéficiaires de l'ASS ont été classés comme RMIstes, le nombre des chômeurs qui retirent un gain net inférieur à 500 francs par mois (ou à $10 \%$ de leurs ressources antérieures) de la reprise d'un emploi à mi-temps est surestimé, et serait de $30 \%$ au lieu de $50 \%$.

\footnotetext{
$\overline{1 . L e s ~ m o d a l i t e ́ s ~ d ' i n t e ́ r e s s e m e n t ~ q u i ~ j o u e n t ~ p e n d a n t ~ u n e ~ d u r e ́ e ~}$ limitée sont négligées.
} 
Tableau 7

Effet de la reprise d'un emploi (pourcentage)

Hausse en pourcentage des ressources nettes des ménages dont un membre non salarié prend un emploi (distribution en pourcentage parmi les chômeurs et inactifs sans salaire déclaré)

\begin{tabular}{|c|c|c|c|}
\hline \multicolumn{4}{|c|}{ Emploi à plein temps rémunéré au Smic } \\
\hline Gain net en \% & Chômeurs & Inactifs & Ensemble \\
\hline Moins de 10 & 2,8 & 4,5 & 3,8 \\
\hline $10-20$ & 13,6 & 18,1 & 16,3 \\
\hline $20-30$ & 13,5 & 11,2 & 12,1 \\
\hline $30-40$ & 10,7 & 13,9 & 12,6 \\
\hline $40-50$ & 29,4 & 17,0 & 21,9 \\
\hline $50-60$ & 12,1 & 9,5 & 10,6 \\
\hline $60-70$ & 7,1 & 5,1 & 5,9 \\
\hline Plus de 70 & 10,8 & 20,8 & 16,8 \\
\hline Total & 100,0 & 100,0 & 100,0 \\
\hline Total (en millions) & 1,9 & 2,9 & 4,9 \\
\hline \multicolumn{4}{|c|}{ Emploi à mi temps rémunéré au Smic } \\
\hline Gain net en \% & Chômeurs & Inactifs & Ensemble \\
\hline Moins de 10 & 53,2 & 41,1 & 45,9 \\
\hline $10-20$ & 15,9 & 21,0 & 18,9 \\
\hline $20-30$ & 20,0 & 17,1 & 18,2 \\
\hline $30-40$ & 6,5 & 3,9 & 4,9 \\
\hline Plus de 40 & 4,5 & 17,0 & 12,0 \\
\hline Total & 100,0 & 100,0 & 100,0 \\
\hline Total (en millions) & 1,9 & 2,9 & 4,9 \\
\hline
\end{tabular}

Source : calculs des auteurs.

Les taux de rétention sont encore plus faibles pour un emploi à mi-temps : la moitié des chômeurs et un tiers des inactifs en retirent moins de 500 francs par mois.

Ces chiffres ne fournissent qu'une image partielle des incitations financières à la reprise d'un emploi. En effet, 3000 francs mensuels, par exemple, peuvent représenter une augmentation très substantielle des revenus du ménage concerné. Tout dépend, en fait, de sa situation individuelle, notamment de la composition familiale et de l'emploi éventuel du conjoint, s'il existe. Pour compléter ces résultats, on a calculé le pourcentage d'accroissement des ressources du ménage quand un de ses membres chômeur ou inactif reprend un emploi payé au Smic. L'accroissement des ressources nettes du ménage lors de la reprise d'un emploi à plein temps au Smic est inférieur à $30 \%$ pour environ un tiers des ménages concernés, que l'individu soit chômeur ou inactif (cf. tableau 7). L'augmentation n'est supérieure à $50 \%$ que pour $30 \%$ des ménages dans le cas d'un chômeur, et $35 \%$ dans le cas d'un inactif. Les chiffres sont bien sûr encore plus spectaculaires dans le cas d'un emploi à mi-temps : $50 \%$ des ménages comprenant un chômeur voient leurs ressources augmenter de moins de $10 \%$ dans ces conditions; seuls $11 \%$ obtiennent une augmentation supérieure à $30 \%$. Une fraction importante de la population concernée (environ $12 \%$ ) n'a pas intérêt financièrement à prendre un emploi à mi-temps rémunéré au SMIC : il s'agit d'individus qui perdant le bénéfice du RMI, se trouvent devenir redevables de la taxe d'habitation et connaissent une réduction brutale de leur allocation logement.

En définitive, l'incitation financière à reprendre un emploi payé au Smic paraît donc faible pour une bonne partie des chômeurs et des inactifs. Afin de préciser ce diagnostic, il faudrait connaître les salaires auxquels peuvent prétendre les personnes en cause, et prendre en compte les aspects non financiers de la décision de participation, liés par exemple à la garde des enfants du ménage. Ceci sort du cadre de cette analyse descriptive mais fait l'objet d'une première exploration dans Laroque et Salanié (1999a ; 1999b). 


\section{BIBLIOGRAPHIE}

Bourguignon F., Chiappori P.-A. et Hugounenq R. (1993), «Explaining the Distribution and Incentive Effects of Tax Harmonization », in A. Heimler et D. Meulders eds, Empirical Approaches to Fiscal Policy Modelling, Chapman-Hall.

CNAF (1996), Prestations Familiales : recettes, dépenses, bénéficiaires. Année 1996. Caisse nationale des allocations familiales, Paris.

Cette G. (1999), Développer le temps partiel choisi, Rapport au Conseil d'analyse économique, Paris.

CSERC (1997), Minima sociaux : entre protection et insertion, Conseil supérieur d'études des revenus et des coûts, Paris.

Groupe Liaisons Sociales (1997), Social pratique : Mémo social 97, Paris.

Hakkio C., Rush M. et Schmidt T. (1996), « The Marginal Income Tax Rate Schedule from 1960 to $1990 »$, Journal of Monetary Economics, $\mathrm{n}^{\circ} 38$, pp. 117-138.

Hourriez J.-M. coordonnateur (1998), « Revenus et patrimoine des ménages », Synthèses, n 19, Insee.

Lang O., Nohrbass K.-H. et Stahl K. (1997), « On Income Tax Avoidance: The Case of Germany », Journal of Public Economics, $n^{\circ}$ 66, pp. 327-347.
Laroque G. et Salanié B. (1999a), «Breaking down Married Female Non-Employment in France », document de travail, $\mathrm{n}^{\circ}$ 9931, Insee-Crest.

Laroque G. et Salanié B. (1999b), « Une décomposition du non-emploi en France », document de travail, $\mathrm{n}^{\circ}$ 9911, Insee-DESE.

Lhommeau B. (1999), « Les configurations familiales des ménages comptant un allocataire du RMI : du foyer RMI au ménage Insee », mimeo, division des Études sociales, Insee.

Mercader-Prats M. (1997), « On the Distributive and Incentive Effects of the Spanish Income Tax: A Comparison of 1980 and $1994 »$, European Economic Review, $\mathrm{n}^{\circ} 41$, pp. 609-617.

Paillaud S. et Eyssartier D. (1998), « Pâris, un outil d'évaluation dynamique du système fiscalo-social », Économie et Statistique, $\mathrm{n}^{\circ} 318$, pp. 41-64.

Piketty T. (1998), « L'impact des incitations financières au travail sur les comportements individuels : une estimation pour le cas français », Économie et Prévision, $\mathrm{n}^{\circ}$ 132-133, pp. 1-35.

Saunier J.-M. coordonnateur (1999), « Les revenus sociaux 1981-1997 », Synthèses, n 21, Insee. 